\title{
Parametric optimization of Liquid Flow Process by ANOVA Optimized DE, PSO \& GA Algorithms
}

\section{Pijush Dutta}

Department of Electronics \& Communication Engineering, Global Institute of Management \& Technology Krishnagar, India, Nadia :741102

pijushdutta009@gmail.com

\section{Madhurima Majumder}

Department of Electrical \& Electronics Engineering, Mirmadan Mohanlal Government Polytechnic, Gobindapur, Plassey, West Bengal 741156

madhurimam128@gmail.com

\section{Asok Kumar}

Dean of student welfare Department, Vidyasagar University, Medinipur, West Bengal, India, Pin: 713305, asok_km650@rediffmail.com

Received: 02 August 2021; Accepted: 15 September 2021; Published: 08 October 2021

\begin{abstract}
Control of liquid level \& flow are the most interest domain in process control industry. Generally process parameter of the liquid flow system is varied frequently during the operation. So the selection of the level of process parameters i.e. input variables seems to be important for achieving the optimum flow rate. In the present work focus is given on the identification of the proper combination of the input parameters in liquid flow rate process. Flow sensor output, pipe diameter, liquid conductivity \& viscosity have been taken as input parameter; flow rate obtained from test is taken as response parameter. Till now several researchers have been performed various optimization methods for optimized the parameters of the process plant. But still computational time \& convergence speed of the applied optimization techniques for the modelling of the nonlinear process system is still an open challenge for the modern research. In this research we proposed three evolutionary algorithms are used to optimize the process parameters of the nonlinear model implemented by ANOVA to mitigate the unbalance, convergence speed and reduce the total computational time. Overall research performed into three stage, in first phase nonlinear equation ANOVA has been used for mathematical model for the process, In second stage three evolutionary algorithms: GA, PSO \& DE are applied for parametric optimization of liquid flow process to maximize the response parameter $\&$ in last phase comparative study performed on simulated results based on confirmed test \& validated our proposed methodology.
\end{abstract}

Index Terms: Liquid Flow model; ANOVA; Particle swarm optimization; differential Evolution; Genetic Algorithm.

\section{Introduction}

Control of liquid flow in process industry is a really difficult task as flow rate of liquid is depending a number of attributes like flow sensor (contact type or non contact type), pipe diameter \& properties of the liquid used in process industry. Generally model of liquid flow control process is a one of the best example of real non linear \& time lagging process. To get the optimal liquid flow there should be proper setting of input parameters otherwise irregular change of input process parameters may cause damage of the whole system.

In modern research a conventional controller used to get optimum flow rate by tuning of input process parameters. The main drawback of this type of conventional controller is very time consuming \& needs a large set of experimental datasets[1]. To overcome this problem a computational intelligent controller used to determine the optimal process parameters for which we get target liquid flow in a process industry. Main advantage of the computational intelligent controller is once the model is designed with the help of training experimental dataset we can easily finding out the value of optimal input process variable for testing output without wasting a large number of datasets \& time lagging. 
There are a number of computational intelligent controllers used in different process industry to achieve the better results. Some of them are predicting of thermal conductivity in a nanofluids [2], predicting in Fault detection of electric switch machine [3] forecasting of load \& energy management system [4], measurement of turbidity [5], liquid flow model [6], prediction of temperature $\&$ discharge of hot water from solar parabolic collector [7], etc. There are a several research has been performed by using different types flow sensor in process industries by taking the consideration of small response time, linearity \& high measuring accuracy. Conventionally in process industries flow rate is measured by the Contact type anemometer [8], Ultrasonic flow meter [9,10], venturi meter [11] etc.

Now the performance of the flow sensor depending upon the number of input variables like the properties of liquid characteristics, pipe diameter, temperature etc. So it is a great challenge how to improve the performance of the flow sensor in process industry. Hence it needed computational algorithm to optimize the process parameters so that calculated output flow rate best fitted with the experimental outcome.

A number researcher has been carried out many Advance control strategies for the liquid flow control process. A feed forward neural network model proposed to predict the liquid flow. A significant accuracy $97.06 \%$ accuracy obtained from this model[12], An optimized fuzzy logic controller (FLC) proposed to achieved the optimum process parameters in anemometer type flow sensor based process system[13]. Highest accuracy obtained from the proposed model was $92.08 \%$ for triangular based membership function. An intelligent FLC proposed to predict liquid flow rate in a given test dataset obtained the maximum accuracy about to 92.28\%[10]. Proposed model utilized in ultrasonic flow sensor based process system. Moreover an empirical model: ANOVA \& RSM was used to make a relation between process output \& input variables from given train dataset obtained from the experimental set up \& finally optimized genetic algorithm used to predict liquid flow rate for a given set of input [14].From the result analysis it was seen that RSM based GA algorithm best fitted the experimental result. An ANN model used to made a non linear relationship between input \& output variables of training result in liquid flow model \& Genetic algorithm used to optimized the process parameters to make the model best fitted [15]. Best accuracy obtained by Rank parent selected GA-ANN model about $98.42 \%$. An ANFIS model investigated to predict the flow rate in flow rate process model [16].Best ANFIS model provides accuracy about $97.857 \%$. An ANN optimized Flower pollination algorithm used to predict the liquid flow model[17]. Maximum accuracy obtained from the testing datasets about $99.25 \%$.Improved versions of original Elephant Swarm Water Search Algorithm (ESWSA) studied the liquid flow control process with non linear, multivariable, boundary conditions \& fluctuation of complex features[18]. An improved hybrid HPSOGWO algorithm proposed to predict the flow rate \& compare the performance with other two algorithm PSO \& GWO[19]. Applied hybrid algorithm outperformed with the accuracy of $99.962 \%$. Hybrid FA-ANN \& PSO-ANN model was proposed to predict liquid flow model[20].

For improving better computational time, precision, convergence speed, better investigation \& proper parameters tuning for taking care of all this, estimation of a highly accurate model for describing a liquid flow control process is still an open problem to us. Due to restriction of process parameter \& interaction between the input process variable $\&$ response it is difficult to obtain the real time quality response from the system. In such cases metaheuristics or bio inspired optimization technique can effectively solve the real time predictive problem for the online measurements. Due to global optimization methods and scale well to higher dimensional problems solving capability here we proposed three different evolution optimization techniques. To represent the non linear model of a liquid flow process here we used mathematical ANOVA.

From the Simulation results it is seen that proposed optimization techniques are effective \& feasible for meet the real-time control requirements of the liquid flow control process. The rest of this paper is organized as follows: after introduction, modeling of the liquid flow control described in section 2 mathematical description is briefly introduced in Section 3. Proposed methodology is described in Section 4 described the results \& discussion and finally conclusions are presented in Section $5 \&$ section 6 respectively.

\section{Experimental Set Up}

The experimental work is carried out with the Flow \& level measurement \& control set up [14](model no. WFT 20-I) shown in Fig .1. For this work, total 134 sample data has been attended which consist of four independent variables sensor output voltage, pipe diameter, liquid (water) conductivity \& viscosity. Among these 17 number of datasets are utilized for the testing purpose shown in Table1.

Table 1.Experimental Testing datasets for liquid flow control process [17]

\begin{tabular}{|l|l|l|l|l|}
\hline Sensor output & Diameter & Conductivity & Viscosity & Flow rate \\
\hline 0.218 & 0.024 & 0.606 & 0.8982 & 0.0008 \\
\hline 0.221 & 0.025 & 0.616 & 0.7797 & 0.0008 \\
\hline 0.225 & 0.025 & 0.616 & 0.8982 & 0.0016 \\
\hline 0.232 & 0.025 & 0.597 & 0.7797 & 0.0016 \\
\hline 0.234 & 0.02 & 0.615 & 0.8982 & 0.0024 \\
\hline 0.237 & 0.027 & 0.622 & 0.7797 & 0.0024 \\
\hline 0.238 & 0.03 & 0.6065 & 0.7254 & 0.0024 \\
\hline
\end{tabular}




\begin{tabular}{|l|l|l|l|l|}
\hline 0.239 & 0.025 & 0.616 & 0.8982 & 0.0032 \\
\hline 0.241 & 0.027 & 0.622 & 0.7797 & 0.0032 \\
\hline 0.245 & 0.024 & 0.6065 & 0.7254 & 0.0032 \\
\hline 0.247 & 0.024 & 0.616 & 0.8982 & 0.004 \\
\hline 0.247 & 0.025 & 0.622 & 0.7797 & 0.004 \\
\hline 0.25 & 0.025 & 0.6065 & 0.7254 & 0.0048 \\
\hline 0.256 & 0.025 & 0.616 & 0.8982 & 0.0048 \\
\hline 0.254 & 0.024 & 0.622 & 0.7797 & 0.0056 \\
\hline 0.259 & 0.03 & 0.606 & 0.7254 & 0.0064 \\
\hline 0.265 & 0.027 & 0.622 & 0.7797 & 0.0072 \\
\hline
\end{tabular}

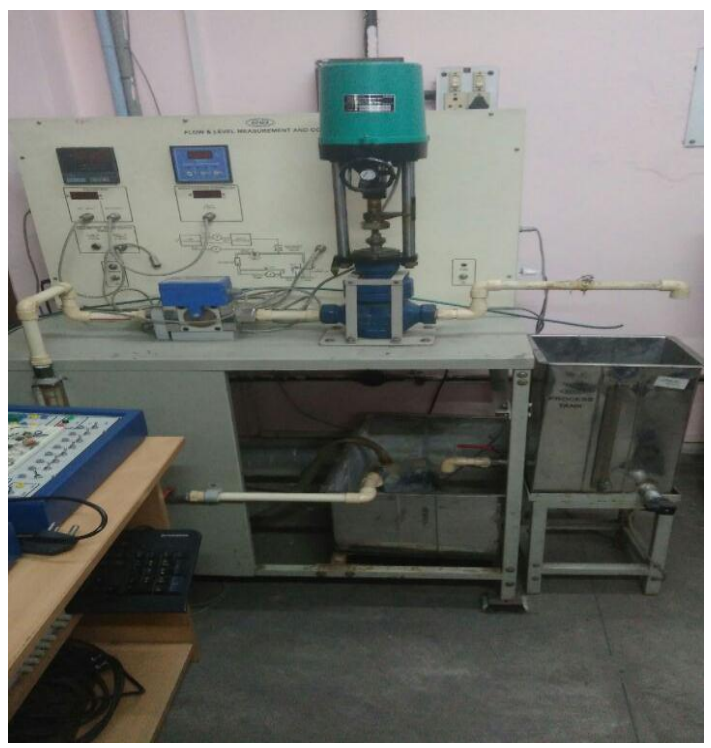

Fig .1. Experimental set up for present research [14]

\section{Modeling of liquid Flow process}

In this section overall methodology of the present research explained. Non linear mathematical equation formulations explained after the flowchart of the research.

\section{A. Flowchart of proposed research}

In present research we formulated a non linear mathematical model: ANOVA from 117 numbers of training datasets. Mathematical formulation of ANOVA explains in later subsection. Three evolutionary algorithms: Genetic Algorithm (GA), Particle swarm Optimization (PSO) \& Differential Evolution (DE) were used finding out the coefficient value of the proposed ANOVA model when objective function reached to optimum. For this purpose we changed the parameters of all mentioned algorithms. Finally we determine the calculated output flowrate corresponding to each set testing input datasets. For the identification of best algorithm in this research we used different statistical metrics like root mean square (RMSE), Mean absolute error (MAE), Mean absolute percentage error (MAPE), accuracy \& computational time etc. Overall process explains in Fig. 2.

\section{B. Mathematical Model}

Due to nonlinear characteristics of liquid flow rate and liquid level process it is very difficult to determine the process input variables like pipe diameter, pipe diameter $\&$ change in liquid properties to achieved the optimum liquid flow rate \& liquid level using conventional controller techniques. Hence we need computational intelligence tools to optimized the non linear mathematical like RSM \& ANOVA [21,22]. In this work, at first we have used popular nonlinear power equations: Analysis of Variance (ANOVA) to describe the mathematical relationship between input variables \& response of liquid flow control process. In the mathematical model flow rate $(F)$ can be expressed in term of sensor output $(E)$, pipe diameter $(D) \&$ liquid properties as follows:

$$
F=\mu_{1} \cdot E^{\mu 2} \cdot D^{\mu 3} \cdot k^{\mu 4} \cdot n^{\mu 5}
$$

Where, $\mu_{1}, \mu_{2}, \mu_{3}, \mu_{4}$ and $\mu_{5}$ are the coefficients the mathematical model. Now computational intelligence techniques are used to get the optimum values of this co efficient from the experimental dataset. Here three different metaheuristics optimization techniques are applied as a computational intelligence tool to achieve the optimum value of the process variable, process fit with the experimental one \& difference between measured and simulated flow rate is 
minimized. So in this research we used RMSE [23] as a objective function for the metaheuristic which is needed to be minimized.

$$
R M S E(X)=\sqrt{\frac{\sum_{i=1}^{N} f(E, D, K, X)^{2}}{N}}
$$

Where, $N$ is the number of the experimental data, $X$ is the set of the estimated parameters.

For ANOVA based modelling, the error function $\left(E_{i}, D_{i}, n_{i}, k_{i}, X\right)$ and set of parameters $X$ can be expressed as

$$
\begin{gathered}
\mathrm{f}\left(E_{i}, D_{i}, n_{i}, k_{i}, X\right)=\mu_{1} \cdot E^{\mu 2} \cdot D^{\mu 3} \cdot k^{\mu 4} \cdot n^{\mu 5}-F \\
X=\left\{\mu_{1}, \mu_{2}, \mu_{3}, \mu_{4}, \mu_{5}\right\}
\end{gathered}
$$

Where, $F$ is the experimental data.
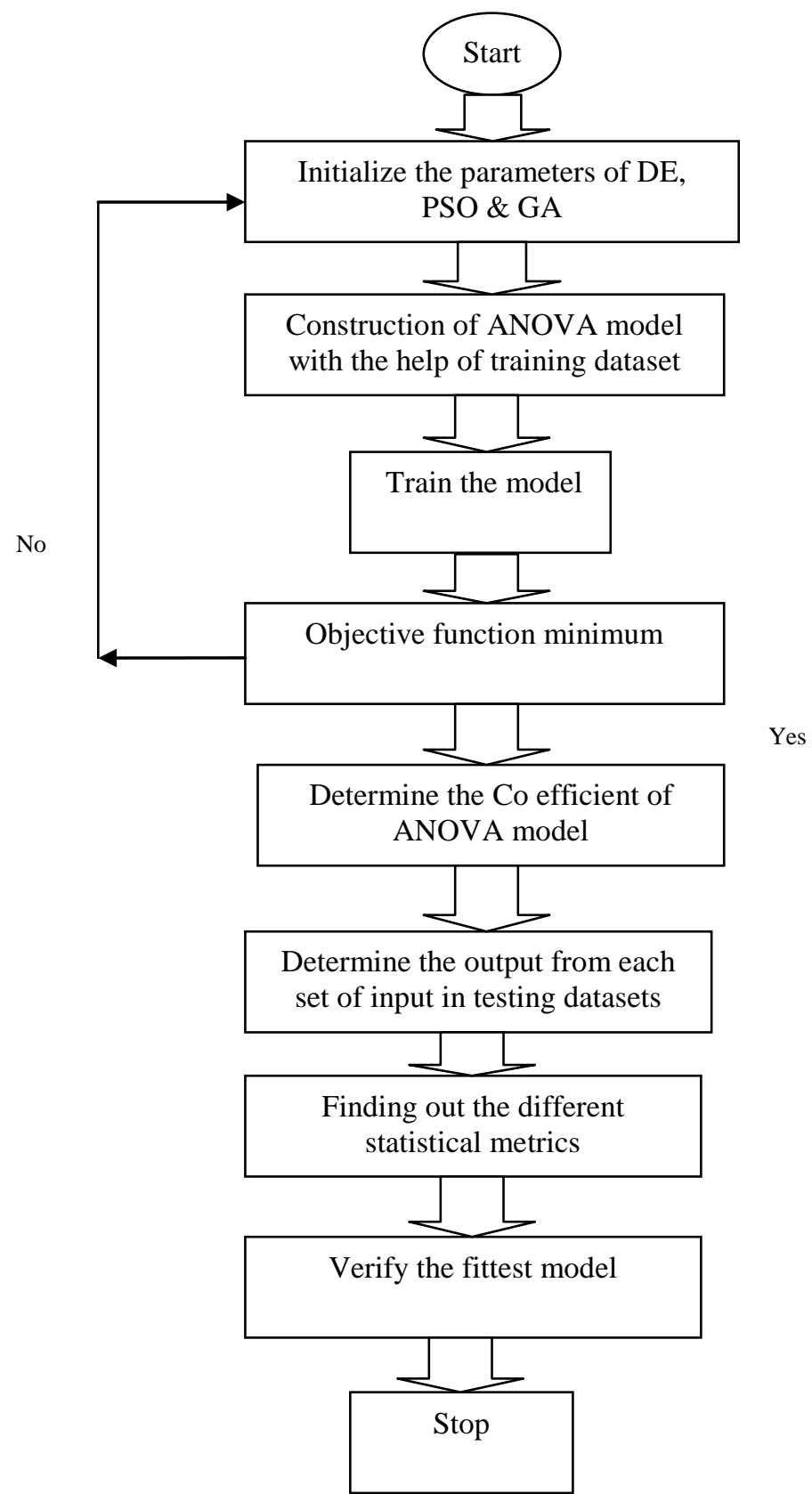

Fig.2. Flowchart for the present research 


\section{Proposed Methodology}

Before elaborating the proposed methodology basic Differential Evolution (DE), Particle Swarm Optimization (PSO) \& Genetic Algorithm (GA) are elaborated initially.

\section{A Differential Evolution calculation $(D E)$}

It is a stochastic, populace based improvement calculation for taking care of nonlinear advancement issue [24]. The calculation was presented by Storn and Price in 1996 [25]. A fundamental variation of the DE calculation works by having a populace of applicant arrangements (called specialists). These specialists are moved around in the inquiry space by utilizing straightforward numerical formulae to join the places of existing operators from the populace [26]. On the off chance that the new position of a specialist is an improvement, at that point it is acknowledged and frames some portion of the populace; generally the new position is just disposed of. The procedure is rehashed and by doing so it is trusted, however not ensured, that a palatable arrangement will inevitably be found[27].

\section{$B$ Particle swarm streamlining (PSO)}

Particle swarm optimization technique is a heuristic technique inspired by the interaction of group of animals[28,29]. The process starts with random variables each of which again represents the possible solution of the optimization problem. Each of the variable represent by two parameters: position \& velocity. Position of the variable indicates the deviation from the ideal optimum solution while the velocity indicates the motion of a variable around the search space. Social performance of PSO depending upon the two important parameters: individual best position $\left(\mathrm{P}_{\text {best }}\right)$ $\&$ global best position $\left(\mathrm{G}_{\text {best. }}\right)$ of each swarm. PSO have been effectively utilized in different research field like: renewable energy[30,31], nanoparticles [32], mathematical modelling [33], nuclear energy field [34] etc.

\section{C.Genetic Algorithm (GA):}

The GA has a place with a group of computational models propelled by Darwin's generation and natural selection hypothesis $[35,36]$. The GA utilizes the essential propagation administrators, for example, hybrid and transformation to deliver the hereditary organization of a populace. Some trade and reordering of chromosomes, creating posterity that contain a mix of data from each parent, is frequently alluded to as hybrid due to the route strands of chromosomes traverse during the trade. Decent variety in the populace is accomplished by change. A regular hereditary calculation methodology makes the accompanying strides: A populace of applicant answers (for the enhancement errand to be tackled) is introduced. New arrangements are made by applying hereditary administrators (change as well as hybrid). The wellness (how great the arrangements are) of the subsequent arrangements is assessed, and reasonable choice procedure is then applied to figure out which arrangements will be kept up into the people to come. The methodology is then iterated. After a few cycles or ages, the calculation unites to the best person that speaks to the ideal answer for the current issue. Hereditary Algorithms have been effectively utilized as an improvement device in operational research[37], multi disciplinary streamlining approach [38,39], the electric vehicle [40], coordination control [41] and aviation applications [42].

\section{Result Analysis}

The parameters setting for every calculation in the examination is portrayed as pursues:

1. For GA, crossover percentage (pc) 0.7 , Extra range factor for crossover $(\gamma) 0.4$, mutation percentage (pm) $0.3 \&$ mutation rate $(\mathrm{mu})$ is 0.1 respectively.

2. For DE, the mutation factor $(\mathrm{F}) 0.5$, crossover rate $(\mathrm{C}) 0.9 \&$ maximum iteration number 200.

3. For PSO, inertia weight $(\mathrm{w}) 1$, inertia weight damping factor $\left(\mathrm{w}_{\mathrm{damp}}\right) 0.99$, personal learning co-efficient $\left(\mathrm{c}_{1}\right) 1.5$ $\&$ Global learning co efficient $\left(c_{2}\right) 2$ according to the earlier work.

For all the algorithms we choose maximum iteration number 5000 and population 100 respectively. For a liquid flow model, search space is confined to 5 dimensional function optimization problems to look through ideal estimations of $\left\{\mu_{1}, \mu_{2}, \mu_{3}, \mu_{4}, \mu_{5}\right\}$ already shown in equation no. 4 . The search range for the optimization of liquid flow based model is $(-15,15)$.

\section{A. Computational Efficiency Test}

Computational time is one of the major criteria for evaluating the effectiveness of the bio inspired optimization technique applied in a particular process parametric optimization. In this subsection we have taken the average execution time taken by each algorithm for each of the problems for a fixed number of iteration 5000, population $100 \&$ 10 times run the program. Table 2 shows a comparative study based on average execution time. From Table 2 It has been observed that GA based ANOVA model performed best by means of average computational time. Graphically visualized 
Table 2 Comparative study based on computational time

\begin{tabular}{|c|c|}
\hline Method & Average computational time \\
\hline DE & $146.1244 \mathrm{Sec}$ \\
\hline PSO & $165.7174 \mathrm{Sec}$ \\
\hline GA & $119.0112 \mathrm{Sec}$ \\
\hline
\end{tabular}

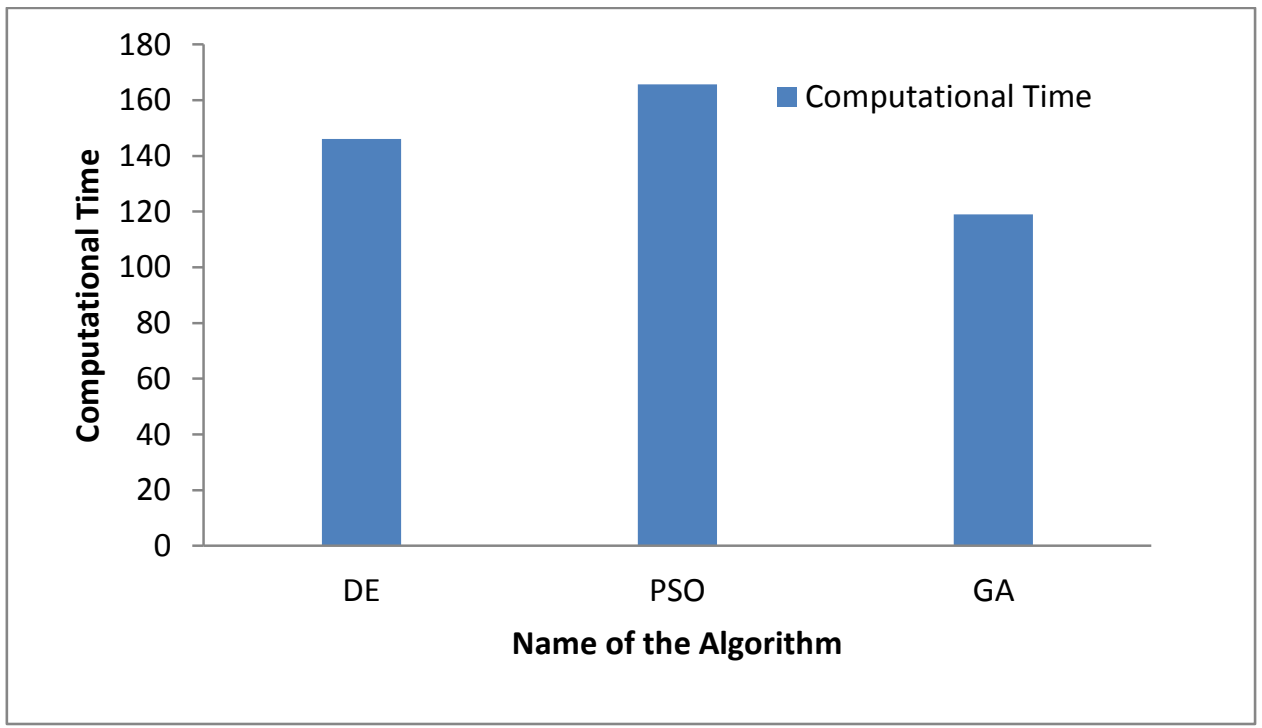

Fig. 3. Comparative study based on Computational time

\section{B. Accuracy Test}

Accuracy test is another statistical criteria which indicates the calculated value is how close to the measured value under the different experimental conditions. In accuracy test we have used two error indicator indexes: Mean absolute error (MAE) \& mean absolute percentage error (MAPE) to measure the error between experimental and the simulated current data, defined as Eqns. (5) and (6)

$$
\begin{gathered}
I A E=\mid \text { Fmeasured }- \text { Fcalculated } \mid \\
\text { MAPE }=\frac{1}{n} \sum_{i=1}^{n} \frac{I A E}{\text { Fmeasured }}
\end{gathered}
$$

Moreover, mean Absolute Error ( $T A E)$ can be defined as:

$$
\mathrm{M} A E=\frac{\sum_{i=1}^{n} I A E_{i}}{n}
$$

Where, $n$ is the number of experimental dataset, Fmeasured \& Fcalculated are the experimental and estimated value of liquid flow rate. The best optimization algorithm always produced least RMSE for all different runs. The coefficient of the non linear models is obtained from DE, PSO \& GA Matlab code shown in Table 3.

Table 3. Estimated optimal parameters by using DE, PSO \& GA based modeling of liquid flow control process

\begin{tabular}{|l|l|l|l|l|l|}
\hline Method & $\boldsymbol{\mu}_{\mathbf{1}}$ & $\boldsymbol{\mu}_{\mathbf{2}}$ & $\boldsymbol{\mu}_{\mathbf{3}}$ & $\boldsymbol{\mu}_{\mathbf{4}}$ & $\boldsymbol{\mu}_{\mathbf{5}}$ \\
\hline DE & 13.9440 & 11.3317 & -1.3593 & -5.0136 & -0.7676 \\
\hline PSO & 15.00 & 10.0466 & -1.0550 & -3.6296 & -0.6247 \\
\hline GA & 15.00 & 8.4074 & -0.7271 & -1.3846 & -0.7300 \\
\hline
\end{tabular}

Table 4. Comparative study based on Mean absolute error (MAE) in DE, PSO \& GA

\begin{tabular}{|c|c|c|}
\hline Method & Mean absolute percentage Error (MAPE) & Mean Absolute Error(MAE) \\
\hline DE & 15.79 & 0.040 \\
\hline PSO & 16.60 & 0.036 \\
\hline GA & 20.41 & 0.039 \\
\hline
\end{tabular}




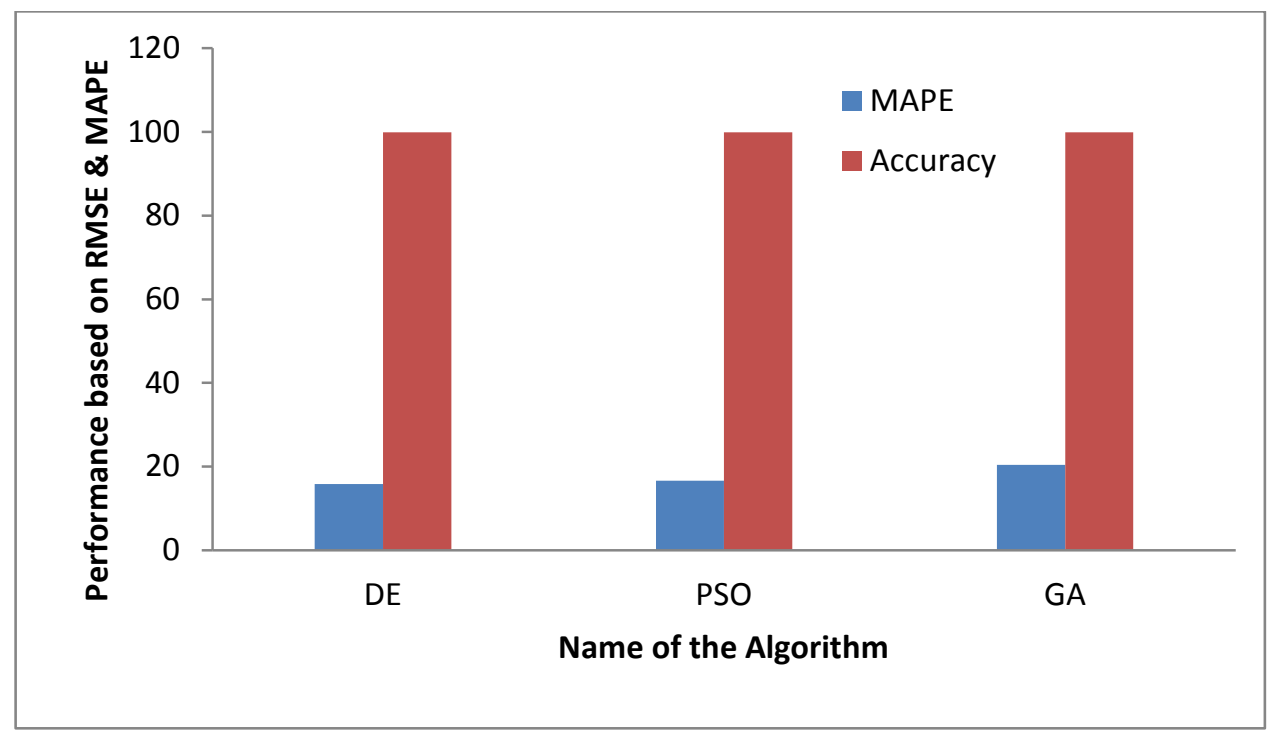

Fig. 4. Comparative study based on MAPE \& Accuracy

The prediction error can be calculated using Root Mean Square Error (RMSE) which can be defined as follows

$$
R M S E=\sqrt{\frac{1}{m} \sum_{i=1}^{m}\left(\frac{X_{\text {exp }}-X_{C a l}}{X_{\text {exp }}}\right)^{2}} * 100 \%
$$

$$
\text { Accuracy }=(100-R M S E) \%
$$

Where, $X_{\text {exp }}$ is experimental value $X_{c a l}$ is calculated value and $m$ is number of training data. From Table 4 describes DE offers least mean absolute Percentage error (MAPE) while PSO gives least mean absolute error (MAE). It has been also observed from Table 5 that PSO optimization has least RMSE error \& maximum accuracy. Fig .6. Show the relative errors vs. different liquid flow rate measurement instances for DE, PSO \& GA based modeling respectively. It can be seen that proposed DE optimization having least relative error than other optimization techniques.

Table 5. Comparative study based on Root mean square error (RMSE) \& Accuracy

\begin{tabular}{|c|c|c|}
\hline Method & RMSE & Accuracy \\
\hline DE & 0.0481 & 99.9519 \\
\hline PSO & 0.0442 & 99.9558 \\
\hline GA & 0.0492 & 99.9508 \\
\hline
\end{tabular}

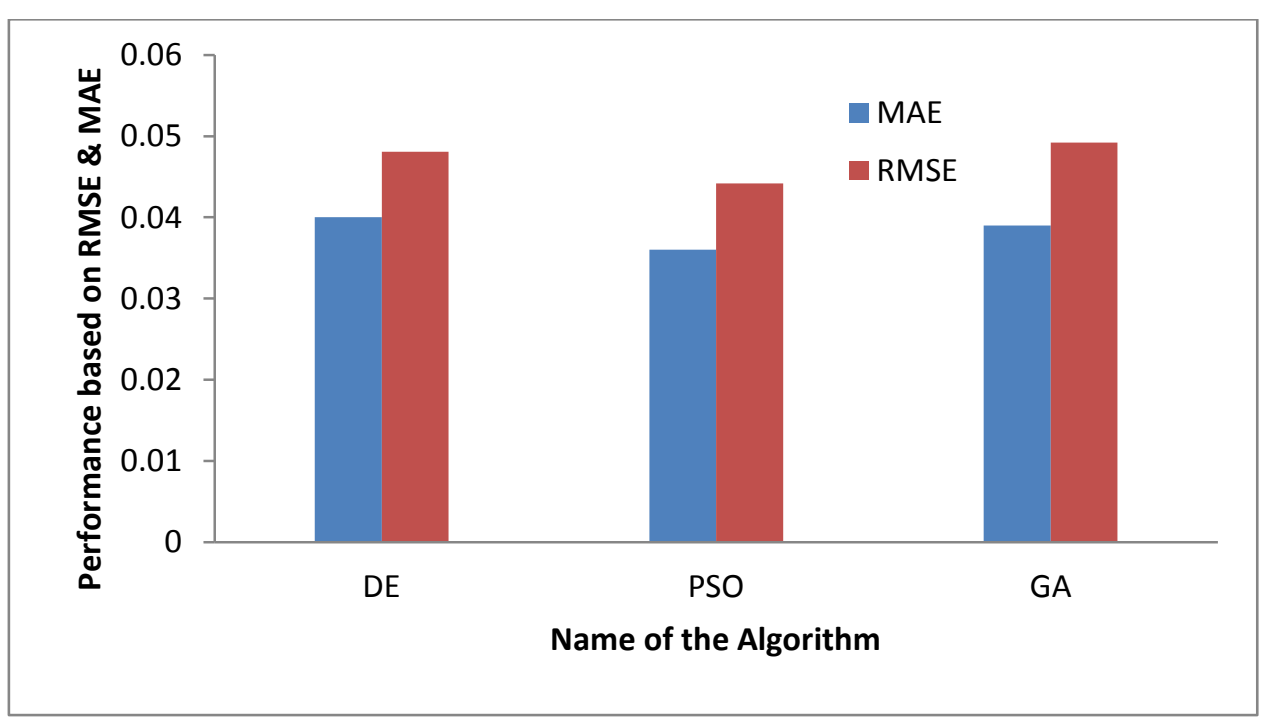

Fig .5. Comparative study based on RMSE \& MAE 
Comparative study between calculated values obtains from GA, DE \& PSO with experimental values of the outputs shown in Fig .7. Characteristics graph represent how all this three evolutionary algorithm calculated flow rate fitted with the experimental flowrate. However among all this EA, GA optimization provides better calculated flowrate with respect to experimental flowrate. Fig. 8.Represent the graph between deviation $\left(=\frac{X_{\exp }-X_{C a l}}{X_{\exp }}\right) \&$ experimental flowrate where the deviation is minimum between the flowrate of $250 \mathrm{Lpm}$ to $500 \mathrm{Lpm}$, DE have least deviation with flowrate.

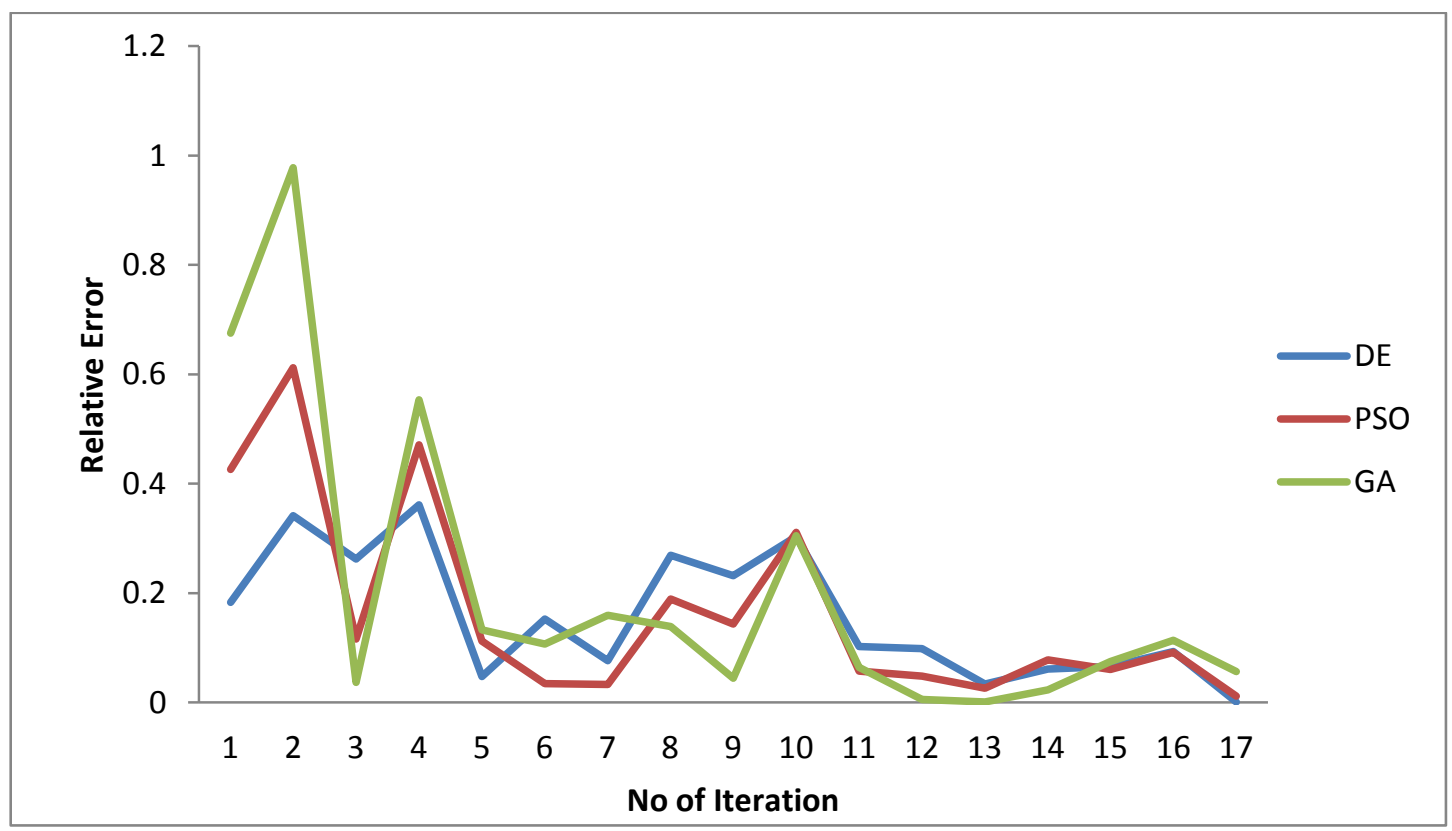

Fig .6. Relative errors for GA, PSO \& DE based modeling of liquid flow control process

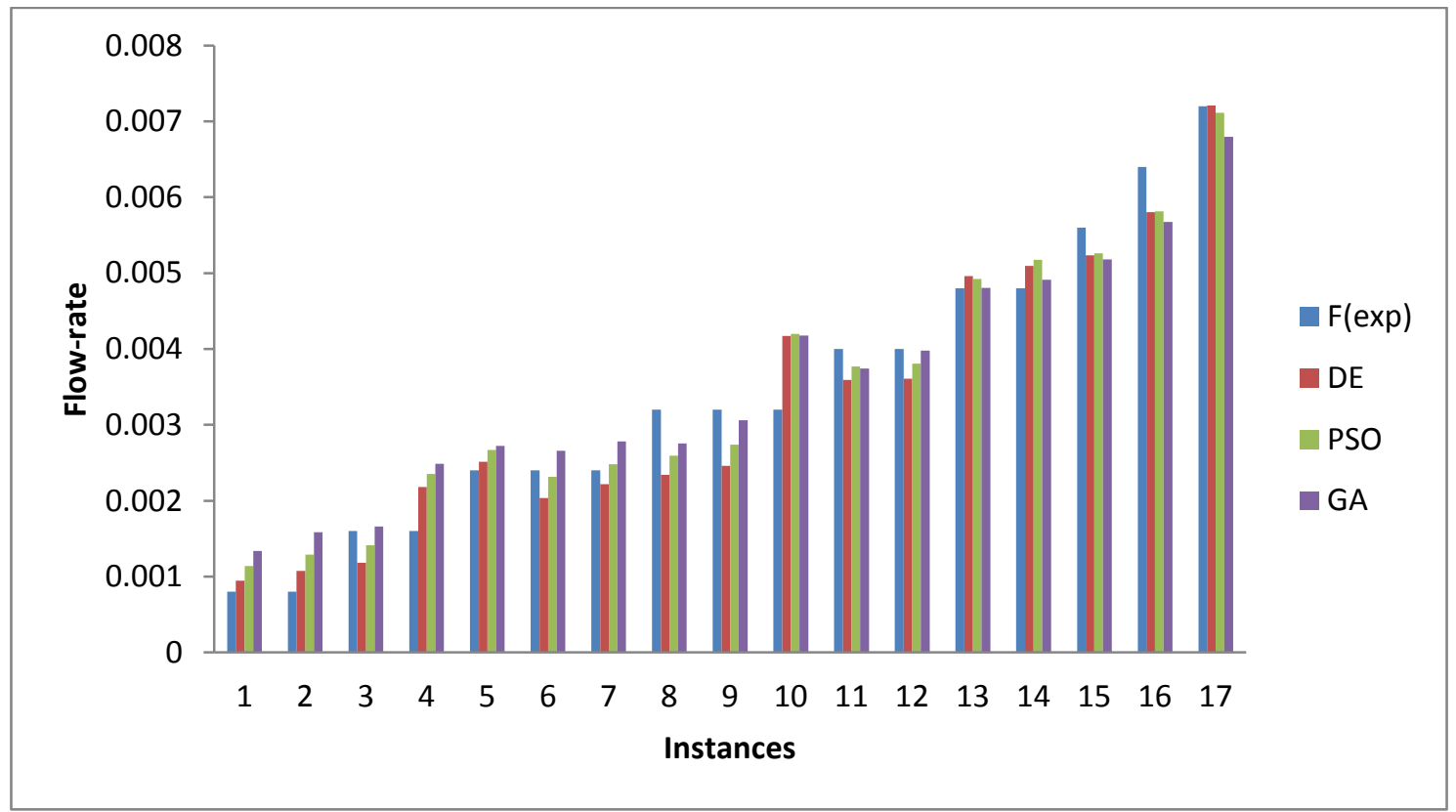

Fig .7. Comparisons of the characteristics of the experimental data and estimated liquid flow rate using DE, PSO \& GA based model 


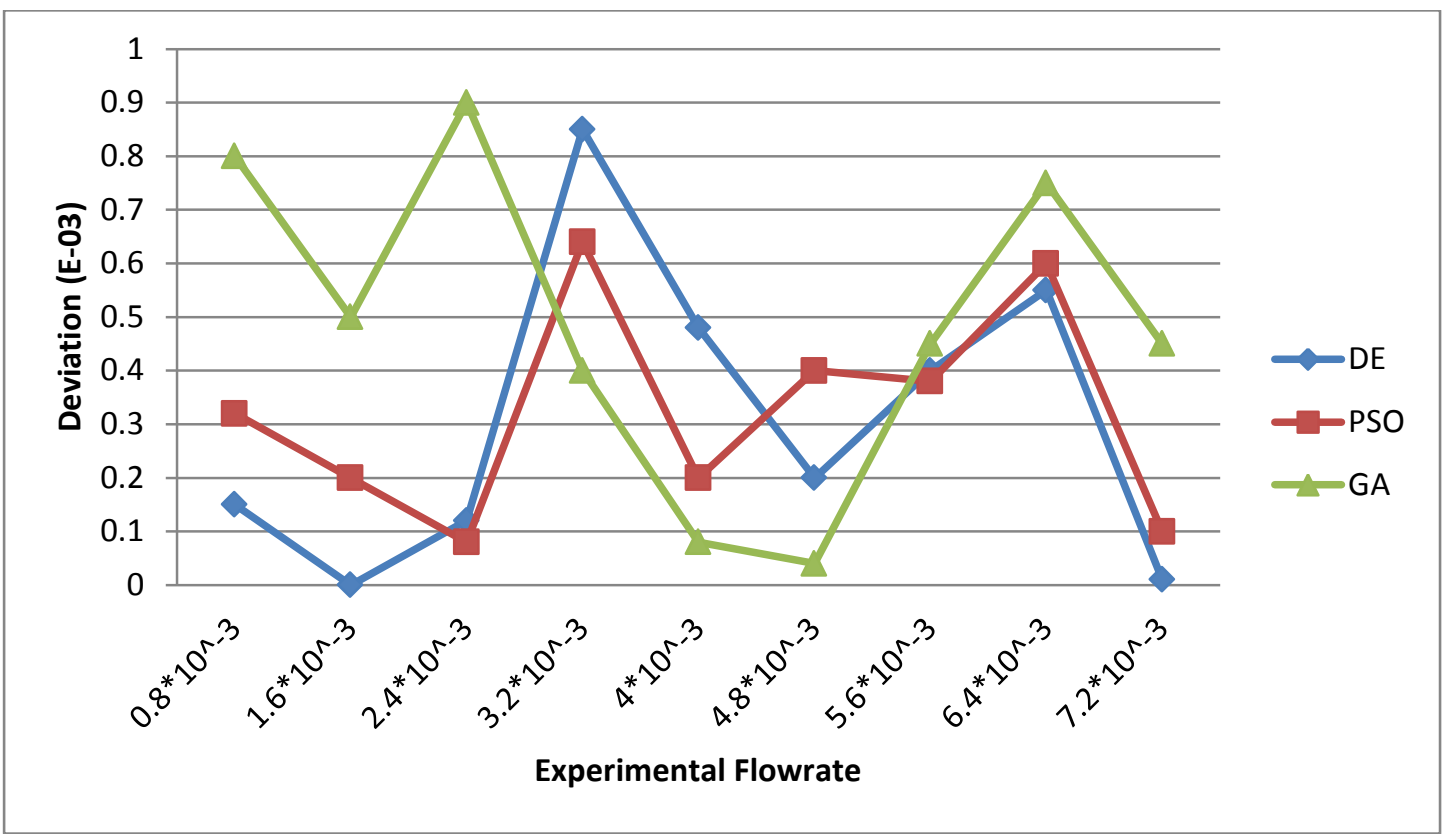

Fig .8. Deviation vs. Experimental flow rate

\section{Score scheme}

To identify the best algorithm among applied three evolutionary algorithms we used score system in present research. Generally scoring system applied in result analysis when a significant variation is taken place between all the proposed algorithms. Here we observed that all the statistical metrics in subsection we cannot conclude which algorithm is better in most of statistical criterion for that we used scoring value in Table 6 .

Table 6. Performance based on scoring scheme

\begin{tabular}{|c|c|c|c|c|c|c|}
\hline Algorithm & RMSE & Accuracy & MAPE & MAE & Computational time & Overall score \\
\hline DE & 1 & 1 & 1.5 & 1 & 1 & 5.5 \\
\hline PSO & 1 & 1 & 1.5 & 1 & 0 & 4.5 \\
\hline GA & 1 & 1 & 0 & 1 & 2 & 5 \\
\hline
\end{tabular}

\section{Conclusions}

Modeling \& optimization of liquid level \& flow control in a process industry is an interesting task for the researchers. In this study, our aim is to optimize the power equation model of ANOVA which is used to make a nonlinear relation between the input $\&$ output of the process variables. In next step we need to find out the optimal values of the coefficient of the ANOVA models using Evolutionary algorithms (DE, PSO \& GA) so that estimated liquid flow rate fit best with the experimental results \& provides the minimum objective function of flow rate. In Final step we perform different statistical analysis which is shown in section 5. From the result analysis we get the following conclusion : in respect to computation time Genetic algorithm is more effective, in respect to MAE, RMSE \& Accuracy PSO show the more effective output \& finally DE shows the better result in respect to MAPE \& Relative error. That's all the evolution algorithm not provides accurate result in all statistical result analysis aspect. However all the algorithms can predict the liquid flow rate with satisfactory accuracy more than $99.95 \%$. From Table 6 It is seen that on the basis of performance score DE algorithm performs better than other two, although there are a small deviation between performance score of mentioned all three algorithms.

More accurate modeling can be designed by considering the other type of contact type liquid flow sensor \& further tuning can be done by metaheuristic optimization techniques to achieve better stability $\&$ accuracy are the future aspect. 


\section{Compliance with ethical standards:}

Conflict of interest: The authors declare that they have no conflict of interests.

\section{References}

[1] Dutta P, Kumar A. A Study on Performance of Different Open Loop PID Tunning Technique for a Liquid Flow Process. IJITCA 2016;6:13-22. https://doi.org/10.5121/ijitca.2016.6202.

[2] Zhang Y, Xu X. Predicting the thermal conductivity enhancement of nanofluids using computational intelligence. Physics Letters A 2020;384:126500. https://doi.org/10.1016/j.physleta.2020.126500.

[3] Soares N, Pestana de Aguiar E, Goliatt L. Failure Classification in Electric Switch Machines Using Symbolic Concepts and Computational Intelligence. 2017. https://doi.org/10.20906/CPS/CILAMCE2017-1021.

[4] Fallah SN, Deo RC, Shojafar M, Conti M, Shamshirband S. Computational Intelligence Approaches for Energy Load Forecasting in Smart Energy Management Grids: State of the Art, Future Challenges, and Research Directions. Energies 2018;11:596. https://doi.org/10.3390/en11030596.

[5] Dutta P, Kumar A. FUZZY MODEL FOR TUBIDITY MEASUREMENT: 4:4.

[6] Dutta P, Kumar A. Effect of Different Defuzzification methods in a Fuzzy Based Liquid Flow control in Semiconductor Based Anemometer. IJITCA 2017;7:01-8. https://doi.org/10.5121/ijitca.2017.7101.

[7] Dutta P, Biswas SK, Biswas S, Majumder M. Parametric optimization of Solar Parabolic Collector using metaheuristic Optimization. Computational Intelligence and Machine Learning 2021;2:7.

[8] DUTTA P, KUMAR A. Flow sensor analogue: realtime prediction analysis using SVM \& KNN, 2018.

[9] Santhosh K, Roy BK. An Intelligent Flow Measurement Technique using Ultrasonic Flow Meter with Optimized Neural Network. 2012:5:4

[10] Dutta P, Kumar A. Intelligent calibration technique using optimized fuzzy logic controller for ultrasonic flow sensor. MATHEMATICAL MODELLING OF ENGINEERING PROBLEMS 2017;4:91-4. https://doi.org/10.18280/mmep.040205.

[11] Roy BK, Venkata SK. A Practically Validated Intelligent Calibration Circuit Using Optimized ANN for Flow Measurement by Venturi | SpringerLink. Journal of The Institution of Engineers (India): Series B 2015:31-9.

[12] DUTTA P, KUMAR A. Study of optimized NN model for liquid flow sensor based on different parameters. Proceeding of international conference on materials, applied physics and engineering, 2018.

[13] Dutta P, Kumar A. Design an intelligent flow measurement technique by optimized fuzzy logic controller. Journal Européen Des Systèmes Automatisés 2018: 51:13: 89-107.

[14] DUTTA P, KUMAR A. Modelling of Liquid Flow control system Using Optimized Genetic Algorithm. Statistics, Optimization \& Information Computing 2020;8:565-82.

[15] DUTTA P, KUMAR A. Design an intelligent calibration technique using optimized GA-ANN for liquid flow control system. Journal Europeen Des Systemes Automatises 2017;50:449-70.

[16] Dutta P, Kumar A. Application of an ANFIS model to Optimize the Liquid Flow Rate of a Process Control System. Chemical Engineering Transactions 2018;71:991-6. https://doi.org/10.3303/CET1871166.

[17] DUTTA P, KUMAR A. Modeling and Optimization of a Liquid Flow Process using an Artificial Neural Network-Based Flower Pollination Algorithm. Journal of Intelligent Systems 2018;29:787-98.

[18] Mandal S, DUTTA P, KUMAR A. Modeling of liquid flow control process using improved versions of elephant swarm water search algorithm. SN Applied Sciences 2019: 1: 8:1-16.

[19] Dutta P, Majumder M, Kumar A. An Improved Grey Wolf Optimization Algorithm for Liquid flow Control System. I J Engineering and Manufacturing 2021;4:10-21

[20] DUTTA P, Cengiz K, KUMAR A. Study of Bio- inspired neural networks for the Prediction of liquid flow in Process control system. Cognitive Big Data Intelligence with a Meta-Heuristic Approach, Elsevier; 2021.

[21] Dutta P, Mandal S, Kumar A. Application of FPA and ANOVA in the optimization of liquid flow control process. RCES 2019;5:7-11. https://doi.org/10.18280/rces.050102.

[22] DUTTA P, Mandal S, KUMAR A. Comparative study: FPA based response surface methodology ANOVA for the parameter optimization in process control. Advances in Modelling and Analysis C 2018;73:23-7.

[23] DUTTA P, Majumder M. AN IMPROVED GREY WOLF OPTIMIZATION TECHNIQUE FOR ESTIMATION OF SOLAR PHOTOVOLTAIC PARAMETERS. International Journal of Power and Energy Systems 2021;41.

[24] Liang J, Xu W, Yue C, Yu K, Song H, Crisalle OD, et al. Multimodal multiobjective optimization with differential evolution. Swarm and Evolutionary Computation 2019;44:1028-59.

[25] Storn R, Ag S, Sn Z, Ring O, Price K. Minimizing the real functions of the ICEC'96 contest by Differential Evolution. IEEE International Conference on Evolutionary Computation (ICEC'96) 1996.

[26] Sun G, Li C, Deng L. An adaptive regeneration framework based on search space adjustment for differential evolution. Neural Computing and Applications 2021:1-17.

[27] Li S, Gu Q, Gong W, Ning B. An enhanced adaptive differential evolution algorithm for parameter extraction of photovoltaic models. Energy Conversion and Management 2020;205:112443.

[28] Bansal JC. Particle swarm optimization. Evolutionary and swarm intelligence algorithms, Springer; 2019, p. 11-23.

[29] Chopard B, Tomassini M. Particle swarm optimization. An Introduction to Metaheuristics for Optimization, Springer; 2018 , p. 97-102.

[30] Khaled U, Eltamaly AM, Beroual A. Optimal power flow using particle swarm optimization of renewable hybrid distributed generation. Energies 2017;10:1013.

[31] Elsheikh AH, Abd Elaziz M. Review on applications of particle swarm optimization in solar energy systems. International Journal of Environmental Science and Technology 2019;16:1159-70. 
[32] Khayati GR. A predictive model on size of silver nanoparticles prepared by green synthesis method using hybrid artificial neural network-particle swarm optimization algorithm. Measurement 2020;151:107199.

[33] Nunes HGG, Pombo JAN, Mariano S, Calado MRA, De Souza JF. A new high performance method for determining the parameters of PV cells and modules based on guaranteed convergence particle swarm optimization. Applied Energy 2018;211:774-91.

[34] Wang H, Peng M, Hines JW, Zheng G, Liu Y, Upadhyaya BR. A hybrid fault diagnosis methodology with support vector machine and improved particle swarm optimization for nuclear power plants. ISA Transactions 2019;95:358-71.

[35] Mirjalili S. Genetic algorithm. Evolutionary algorithms and neural networks, Springer; 2019, p. 43-55.

[36] Lambora A, Gupta K, Chopra K. Genetic algorithm-A literature review. 2019 International Conference on Machine Learning, Big Data, Cloud and Parallel Computing (COMITCon), IEEE; 2019, p. 380-4.

[37] Metawa N, Hassan MK, Elhoseny M. Genetic algorithm based model for optimizing bank lending decisions. Expert Systems with Applications 2017; 80:75-82.

[38] Kor O, Acarer S. Aerodynamic Optimization of a Compressor Rotor Using Genetic Algorithm. Designing Engineering Structures Using Stochastic Optimization Methods, CRC Press; 2020, p. 187-202.

[39] Sharma R, Jha BK, Pahuja V. Optimization Techniques for Response Predication in Metal Cutting Operation: A Review. Proceedings of the International Conference on Industrial and Manufacturing Systems (CIMS-2020), Springer; 2022, p. 77-92.

[40] Zou S, Lu J, Mallik A, Khaligh A. Modeling and optimization of an integrated transformer for electric vehicle on-board charger applications. IEEE Transactions on Transportation Electrification 2018; 4:355-63.

[41] Zhang Y, Zhou Y. Distributed coordination control of traffic network flow using adaptive genetic algorithm based on cloud computing. Journal of Network and Computer Applications 2018; 119:110-20.

[42] Tran HK, Nguyen TN. Flight motion controller design using genetic algorithm for a quadcopter. Measurement and Control $2018 ; 51: 59-64$.

\section{Authors' Profiles}

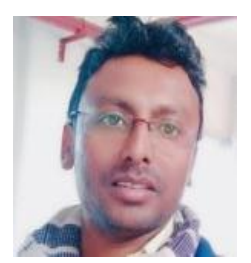

Prof. Pijush Dutta is currently working as an Assistant Professor in Department of Electronics \& communication Engineering, Global Institute of Management \& Technology, India. He received his B Tech \& M Tech from West Bengal University of Technology, India in 2007 \& 2012 respectively. Presently he Persuing his $\mathrm{Ph} \mathrm{D}$ from Mewar University, India. Till now he published more than 38 research journal, Conference \& Book chapter, 2 author book \& 14 internal \& national Patent. His research interests are optimization, intelligent system, Internet of Things, Machine Learning etc.

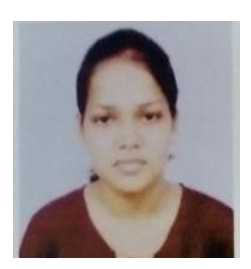

Mrs. Madhurima Majumder is currently acting as a lecturer in Department of electrical \& Electronics Engineering Department, Mirmadan Mohanlal Government Polytechnic Institute, India. She received her B.Tech from MAKAUT, India in 2019. Presently she is Persuing M Tech from MAKAUT. Till now she published 5 research papers in a reputated journal. Her research interests are Internet of Things \& Artificial Intelligence.

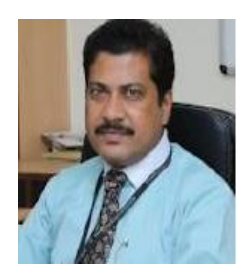

Prof. (Dr.) Asok Kumar is currently working as a Dean of Student welfare Department, Vidya- Sagar University, India. He received his M.Tech from Calcutta University \& Ph D Degree from Jadavpur University in 1999 \& 2007 respectively. Till now he published more than 70 reputated journal, conference, \& book chapter and 2 author book. His research interests are networking, coding techniques, intelligent system etc.

How to cite this paper: Pijush Dutta, Madhurima Majumder, Asok Kumar, " Parametric optimization of Liquid Flow Process by ANOVA Optimized DE, PSO \& GA Algorithms ", International Journal of Engineering and Manufacturing (IJEM), Vol.11, No.5, pp. 14-24, 2021. DOI: 10.5815/ijem.2021.05.02 